\title{
Pronóstico de precipitaciones mensuales: Un estudio de caso en las comunidades nativas del Perú
}

\section{Forecasting monthly rainfall: A case study in Peruvian native communities}

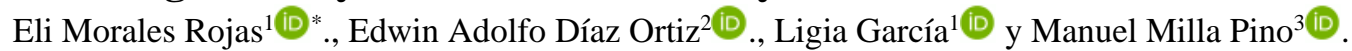

\section{RESUMEN}

El análisis de registros de precipitación permite determinar patrones de cambio actuales y retrospectivos, que usados adecuadamente determinarán el potencial de agua de lluvia. El objetivo de la investigación fue pronosticar las precipitaciones mensuales, para la evaluación de instalación de sistemas de captación de agua de lluvia en comunidades nativas de Perú. Se evaluó el comportamiento de las precipitaciones durante los años 2016-2020 con datos del SENAMHI y se determinó los pronósticos de precipitación para el año 2021 y 2022. Se utilizó el modelo aditivo de Holt-Winters para el cálculo de las predicciones. La precipitación promedio durante los 5 años evaluados fue 1786.24 (mm). El área promedio del techo de las viviendas fue de $47.5 \mathrm{~m}^{2}$, la cantidad de captación de agua fue de 76361.76 litros anuales/vivienda. Las estimaciones de los pronósticos son constantes, el modelo de tendencia cuadrática arrojó que, para el año 2021 la precipitación se estima en 2 038,804 (mm) y para el año 2022 se estiman 2217.338 (mm).

Palabras clave: Agua de lluvia, tendencias, pronósticos, precipitación.

\begin{abstract}
The analysis of rainfall records allows current and retrospective patterns of change to be determined, which when used appropriately will determine rainwater potential. The objective of the research was to forecast monthly rainfall for the evaluation of the installation of rainwater harvesting systems in native communities in Peru. The behaviour of rainfall during the years 2016-2020 was evaluated with data from SENAMHI and rainfall forecasts for the years 2021 and 2022 were determined. The Holt-Winters additive model was used to calculate the forecasts. The average precipitation during the 5 years assessed was $1786.24(\mathrm{~mm})$. The average roof area of the dwellings was $47.5 \mathrm{~m} 2$, the amount of water catchment was 76361.76 litres per year/household. The forecast estimates are constant, the quadratic trend model yielded that, for the year 2021 the rainfall is estimated at 2038.804 (mm) and for the year 2022 it is estimated at $2217.338(\mathrm{~mm})$.
\end{abstract}

Keywords: Rainfall, trends, forecasts, precipitation.

DOI: https://doi.org/10.37787/pakamuros-unj.v9i3.219

Recibido: 28/05/2021. Aceptado: 01/07/2021

* Autor para correspondencia

1. $\quad$ Escuela de Postgrado de la Universidad Nacional Toribio Rodríguez de Mendoza de Amazonas, Chachapoyas 01001, Perú. Email: eli.morales.epg@untrm.edu.pe

2. Facultad de Ingeniería Civil y Ambiental, Universidad Nacional Toribio Rodríguez de Mendoza de Amazonas, Perú. Email: edwin.diaz@untrm.edu.pe

3. Facultad de Ingeniería Civil. Universidad Nacional de Jaén (UNJ), Jirón Cuzco, n 250, 06801, Jaén, Cajamarca, Perú., País. Email: manuel.milla@unj.edu.pe 


\section{INTRODUCCIÓN}

La precipitación es importante en el ciclo hidrológico, por ser la cantidad de agua que cae a la superficie terrestre y provee de agua dulce para el desarrollo de la vida (Priyan, 2015). Las precipitaciones son importantes conocerlas, ya sea para la prevención de riesgos y diferentes actores, tales como los sectores productivos y económicos (Olivares et al., 2016). Son de gran utilidad para evaluar el comportamiento del clima y pronosticar efectos sobre la disponibilidad futura de agua, excepcionalmente en las comunidades nativas (González-Reyes \& Muñoz, 2013).

A través de las tendencias de regresión lineal anuales y estacionales se puede analizar el aumento o la disminución de las precipitaciones durante un periodo proyectado (Márdero et al., 2012). Es importante las estimaciones, dado que ayuda a pronosticar, a medida que la atmósfera terrestre se calienta, y no se sabe cómo cambiará la precipitación, ni cómo estos cambios afectarán las precipitaciones regionales (Magallanes-Quintanar et al., 2019). Con la implantación de modelos de predicción, se determina las características de zonas climatológicas con diferentes intensidades (Siles \& Chirinos, 2018).

La predicción en temas medioambientales hace que utilicen métodos estadísticos más precisos para estudiar el tiempo y el cambio climático, entre ellos está el modelo de Holt-Winters, el cual puede predecir variables de temperatura y precipitación (Heydari et al., 2020). El modelo de Holt Winters es usado para pronosticar y estimar parámetros del tiempo (Liu \& Wu, 2020; Puah et al., 2016; Shah et al., 2018).

En Tailandia utilizaron el modelo de Holt Winters para estimar precipitaciones, afianzándose el error porcentual medio (MAPE) y el error medio absoluto (MAE) (Sopipan, 2014). Las precipitaciones muestran una alta variabilidad, según los análisis realizados mediante un análisis de frecuencia, donde los valores pueden ser crecientes o decrecientes según las épocas del año (Wong, 2009). En Irán demostraron que los datos de severidad de la lluvia tuvieron altas variaciones a los valores promedio (Tabari et al., 2011). Las estimaciones de las precipitaciones en los últimos 5 años han aumentado, dado la importancia, y permita la toma de decisiones adecuadas basados en predicciones (Tabla 1).

Tabla 1. Investigaciones en la aplicación del modelo Holt Winters en los últimos 5 años

\begin{tabular}{cccc}
\hline Cita & Tipo modelo Holt-Winters & Aplicación & País \\
\hline Sinay \& Kembauw, 2021 & Exponencial & Predicción de & Indonesia** \\
& & precipitaciones & \\
& & \\
\hline
\end{tabular}




\begin{tabular}{|c|c|c|c|}
\hline Shah et al., 2018 & Aditivo y multiplicativo & $\begin{array}{l}\text { Predicción de } \\
\text { precipitaciones }\end{array}$ & India*** \\
\hline Manideep \& Sekar, 2018 & Aditivo y multiplicativo & $\begin{array}{l}\text { Predicción de } \\
\text { precipitaciones }\end{array}$ & India*** \\
\hline Mohammad et al., 2021 & Multiplicativo & $\begin{array}{l}\text { Predicción de } \\
\text { precipitaciones }\end{array}$ & Inglaterra* \\
\hline $\begin{array}{l}\text { Dhamodharavadhani \& } \\
\text { Rathipriya, } 2019\end{array}$ & Exponencial & $\begin{array}{l}\text { Predicción de } \\
\text { precipitaciones }\end{array}$ & India*** \\
\hline Raha \& Gayen, 2021 & Aditivo y multiplicativo & Predicción de sequias & India*** \\
\hline Pushpalatha, 2019 & Exponencial & Predicción de sequias & India*** \\
\hline Anggono et al., 2018 & Aditivo & Predicción de sequias & Indonesia** \\
\hline Salauddin Khan et al., 2018 & Exponencial & $\begin{array}{c}\text { Predicción de } \\
\text { precipitaciones } \\
\text { temperaturas, humedad y }\end{array}$ & Bangladés* \\
\hline
\end{tabular}

*un estudio; **dos estudios; ***más de tres estudios

En base a lo mencionado el objetivo fue determinar las tendencias de precipitación mensual, con fines de instalación de sistemas de captación de agua de lluvia, en las comunidades nativas de Tunants y Yahuahua.

\section{MATERIALES Y MÉTODOS}

La zona de estudio estuvo localizada en las comunidades nativas de Tunants y Yahuahua de la selva del Perú, departamento Amazonas, provincia Condorcanqui, distrito Nieva. Las coordenadas se tomaron con GPS modelo GPSMAP 66i-GARMIN (Tabla 1 y Figura 1).

Tabla 1. Coordenadas de la ubicación de las comunidades

\begin{tabular}{|c|c|c|c|c|}
\hline \multirow[b]{2}{*}{ Comunidades } & \multicolumn{2}{|c|}{ Coordenadas } & \multirow[t]{2}{*}{ Altitud (msnm) } & \multirow[t]{2}{*}{ Temperatura $\left({ }^{\circ} \mathrm{C}\right)$} \\
\hline & Este & Norte & & \\
\hline \multirow{2}{*}{ Tunants } & 830543 & 9481810 & & \\
\hline & 830880 & 9481944 & 196 & 26 \\
\hline \multirow{2}{*}{ Yahuahua } & 832070 & 9482801 & & \\
\hline & 167331 & 9482999 & & \\
\hline
\end{tabular}




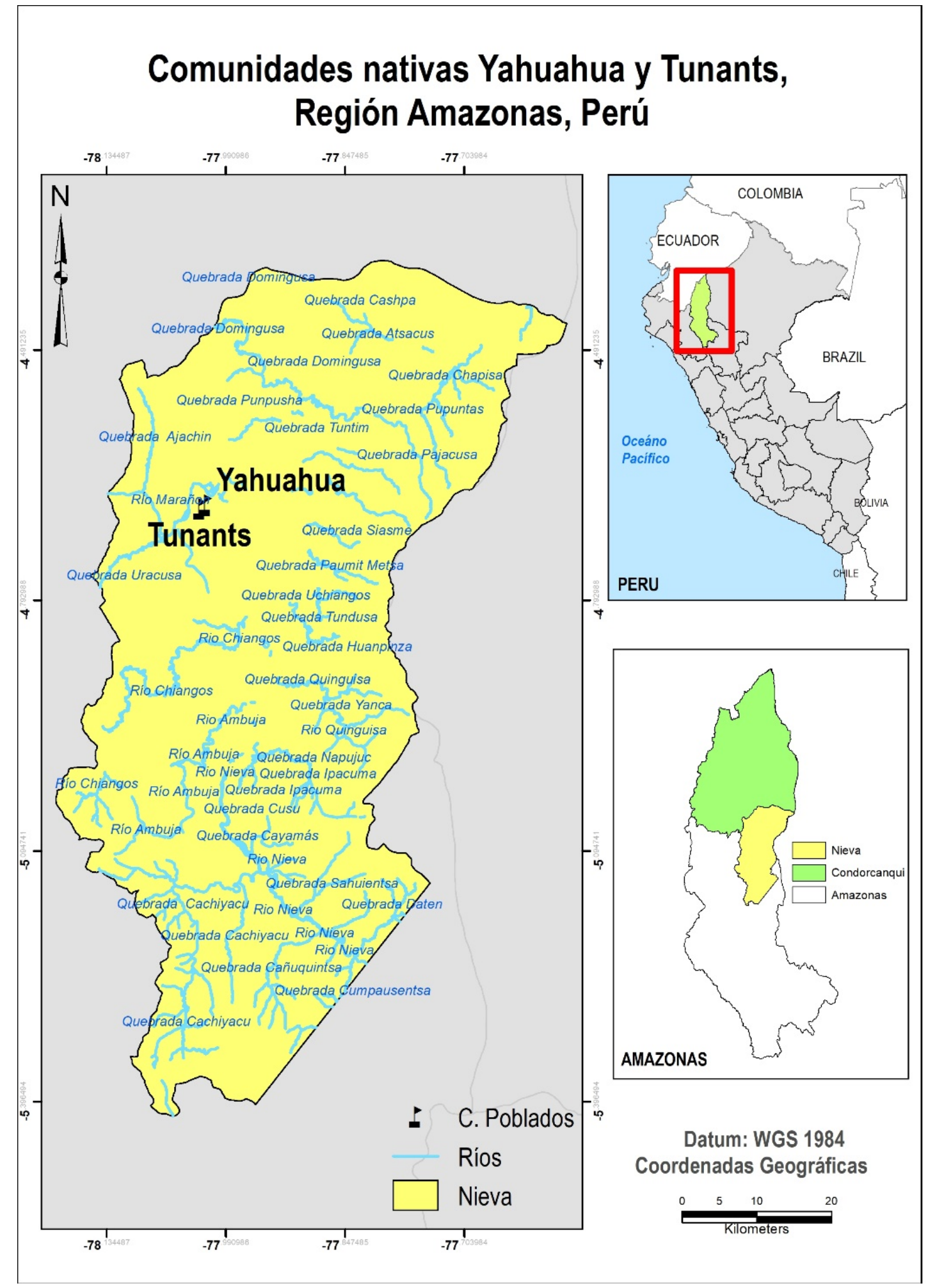

Figura 1. Mapa de ubicación de las comunidades nativas de Tunants y Yahuhua 
Estas comunidades no cuentan con abastecimiento de agua potable destinado al consumo humano. Se abastecen de agua, proveniente del río marañón y lagos cercanos (Tunants y Yahuahua).

Comúnmente el agua lo acarrean y lo depositan en cilindros o baldes grandes, lo dejan reposar mínimo media hora para su uso (Figura 2).

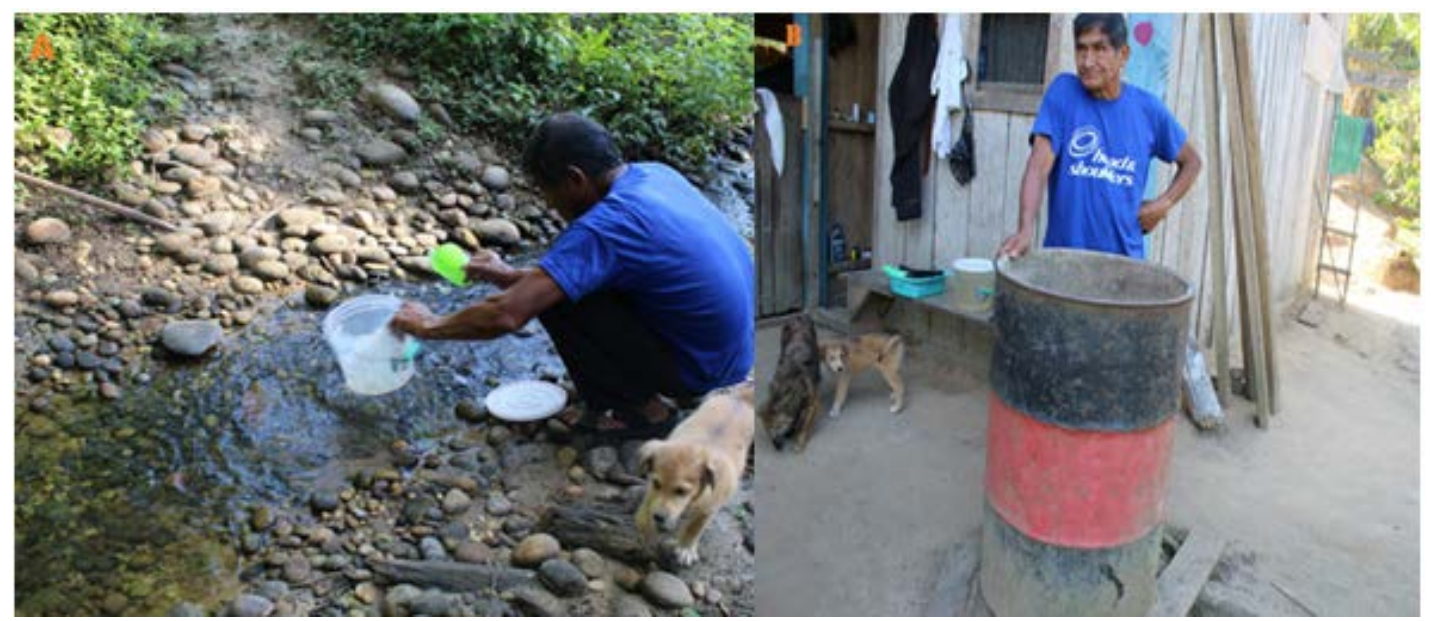

Figura 2. Nativo recolectando agua de lluvia (A); Cilindro de almacenamiento de agua (B)

\section{Estimación de la captación de agua de lluvia por vivienda}

La colecta de datos de precipitación se realizó de la estación del Servicio Nacional de Meteorología e Hidrología del Perú (SENAMHI) y se evaluó el comportamiento de las precipitaciones desde el 2016 al 2020 (máximos, mínimos y desviación estándar), así como la captación promedio de agua de lluvia por vivienda, destinado al consumo humano, se muestra en la ecuación (1).

$$
\text { Allca }=\text { Precipitación } * A C * C_{e}
$$

Donde:

Allca=Agua de lluvia captada.

$A C=$ Área de captación (Tamaño de techo de la vivienda, puede ser variable).

$C e=$ coeficiente de escorrentía (Lámina metálica galvanizada R.M. № 173-2016-VIVIENDA).

\section{Modelo Holt-Winters aditivo}

El modelo aditivo se determinó en base a las ecuaciones (1), (2), (3) y (4): (Sinay \& Kembauw, 2021).

\section{Nivel estimado:}




$$
B_{s}=\propto\left(X_{t}-S_{t-S}\right)+(1-\propto)\left(X_{t-1}+T_{t-1}\right)
$$

Determinación de la tendencia:

$$
T_{t}=\gamma\left(B_{s}-B_{s-1}\right)+(1-\gamma) T_{t-1}
$$

Determinación de la estacionalidad:

$$
S_{t}=\delta\left(X_{t}-B_{S}\right)+(1-\delta) S_{t-1}
$$

Determinación de periodos futuros

$$
\hat{X}_{t+m}=B_{s}+m T_{T}+S_{T+m-s}
$$

\section{Modelo Holt-Winters multiplicativo}

El modelo multiplicativo se basó en las ecuaciones 1, 2, 3 y 4.

Nivel estimado:

$$
B_{s}=\propto \frac{X_{t}}{S_{t-s}}+(1-\propto)\left(B_{s-1}+T_{t-1}\right)
$$

Determinación de la tendencia:

$$
T_{t}=\gamma\left(B_{s}-B_{s-1}\right)+(1-\gamma) T_{t-1}
$$

Determinación de la estacionalidad:

$$
S_{t}=\delta \frac{X_{t}}{B_{s}}+(1-\delta) S_{t-s}
$$

\section{Determinación de periodos futuros}

$$
\hat{X}_{t+m}=B_{s}+m T_{T}+S_{T+m-s}
$$

Donde:

$B_{S}$ : Es el valor suavizado en un periodo.

$\alpha$ : Es la constante de suavización exponencial para la tendencia.

$\gamma$ : Es la tendencia de suavización para la estacionalidad. 
$s$ : Es el tiempo de la estacionalidad.

$m$ : Es los periodos futuros.

$\widehat{X}_{t+m}:$ es la prediccion del modelo Holt - Winters

\section{Elección del modelo para estimación de las precipitaciones}

El modelo se eligió en función Error Porcentual Absoluto Medio (MAPE), en ese sentido se ha procedido a evaluar el modelo que tenga menor MAPE, comparándole entre el modelo Holt-Winters aditivo y HoltWinters multiplicativo (Puah et al., 2016; Wichitarapongsakun et al., 2016). También se evaluó la desviación cuadrática media (MSD) que está relacionada con los valores del MAPE. El cálculo del MAPE, MAD y el MSD se realizó mediante la estadística descriptiva, con la aplicación de series de tiempo. La verificación se realizó mediante las constantes de suavización $\alpha$ (nivel) $0.2, \gamma$ (tendencia) 0.2 $\delta$ (estacional) 0.2. Siendo el mejor modelo ajusta el Holt-Winters aditivo (Tabla 2).

Tabla 2. Indicadores para el modelo de Holt-Winters aditivo y el modelo Holt-Winters multiplicativo

\begin{tabular}{ccccccc}
\hline & \multicolumn{3}{c}{ Holt-Winters aditivo } & \multicolumn{2}{c}{ Holt-Winters multiplicativo } \\
\cline { 2 - 6 } Meses & MAPE & MAD & MSD & MAPE & MAD & MSD \\
\hline Enero & $13.25^{*}$ & 17.33 & 332.08 & $13.19^{* *}$ & 17.15 & 324.82 \\
Febrero & $51.80^{* *}$ & 66.79 & 5835.83 & $55.41^{*}$ & 73.72 & 7117.91 \\
Marzo & $14.65^{*}$ & 23.54 & 658.65 & $14.09^{* *}$ & 23.21 & 692.79 \\
Abril & $64.08^{*}$ & 57.23 & 5838.49 & $55.78^{* *}$ & 49.72 & 4299.24 \\
Mayo & $48.62^{* *}$ & 59.11 & 3811.71 & $49.99^{*}$ & 61.35 & 4079.76 \\
Junio & $6.63^{* *}$ & 8.73 & 122.32 & $8.68^{*}$ & 11.58 & 178.15 \\
Julio & $26.87^{*}$ & 33.38 & 1147.82 & $26.21^{* *}$ & 32.63 & 1093.49 \\
Agosto & $90.07^{* *}$ & 63.40 & 5894.61 & $90.55^{*}$ & 62.24 & 5655.34 \\
Setiembre & $20.45^{* *}$ & 20.17 & 477.45 & $21.25^{*}$ & 21.67 & 537.05 \\
Octubre & $4.80^{* *}$ & 5.78 & 41.01 & $4.87^{*}$ & 5.89 & 42.17 \\
Noviembre & $30.42^{* *}$ & 44.61 & 2182.24 & $32.82^{*}$ & 45.11 & 2239.59 \\
Diciembre & $37.36^{* *}$ & 52.80 & 5320.45 & $37.53^{*}$ & 52.54 & 5680.87 \\
Total & $408.99^{* *}$ & 452.88 & 31662.65 & $410.36^{*}$ & 456.81 & 31941.17 \\
\hline
\end{tabular}

**Ajuste con menor error porcentual absoluto medio para la toma de decisión; *Ajusta con mayor error porcentual absoluto medio; MAPE=Error Porcentual Absoluto Medio; MAD=Desviación absoluta de la media; MSD= Desviación cuadrática media.

\section{Análisis de datos}

El análisis de datos para la estimación de las precipitaciones, así como la determinación de los máximos y mínimos se realizó mediante el software estadístico minitab 2019 (Okagbue et al., 2021). 


\section{RESULTADOS}

Las precipitaciones muestran diferencias entre los valores máximos y mínimos, y tienen relación con las épocas del año (lluviosas y estiaje) (Tabla 3).

Tabla 3. Mínimos y máximos de la precipitación de agua de lluvia

\begin{tabular}{cccccc}
\hline $\begin{array}{l}\text { Estadístico } \\
\text { descriptivo }\end{array}$ & $\mathbf{2 0 1 6}$ & $\mathbf{2 0 1 7}$ & $\mathbf{2 0 1 8}$ & $\mathbf{2 0 1 9}$ & $\mathbf{2 0 2 0}$ \\
\hline Máximo $(\mathrm{mm})$ & $189.9^{\text {mayo }}$ & $201.9^{\text {marzo }}$ & $230.9^{\text {diciembre }}$ & $260^{\text {febrero }}$ & $169.6^{\text {febrero }}$ \\
Mínimo $(\mathrm{mm})$ & $92.8^{\text {abril }}$ & $59.4^{\text {agosto }}$ & $92.4^{\text {setiembre }}$ & $129.3^{\text {febrero }}$ & $54.6^{\text {agosto }}$ \\
Promedio $(\mathrm{mm})$ & $143.84 \pm 33.40$ & $114.70 \pm 43.50$ & $135.10 \pm 43.50$ & $185.65 \pm 43.50$ & $113.69 \pm 43.50$ \\
\hline
\end{tabular}

Tabla 4, se muestra los resultados de captación de agua de lluvia en el área de techo de $47.5 \mathrm{~m}^{2}$, utilizando los pronósticos del modelo Holt- Winters aditivo, durante el año 2021-2022.

Tabla 4. Captación de agua de lluvia mediante modelo Holt- Winters aditivo, para el año 2021 y 2022

\begin{tabular}{|c|c|c|c|c|}
\hline \multirow{3}{*}{ Meses del año } & \multicolumn{4}{|c|}{ Agua cosechada, mediante modelo Holt- Winters aditivo } \\
\hline & \multicolumn{2}{|c|}{ Año 2021} & \multicolumn{2}{|c|}{ Año 2022} \\
\hline & Litros & $\mathbf{m}^{3}$ & Litros & $\mathbf{m}^{3}$ \\
\hline 01 & 5786.51 & 5.79 & 6514.33 & 6.51 \\
\hline 02 & 11123.51 & 11.12 & 13040.67 & 13.04 \\
\hline 03 & 8122.63 & 8.12 & 6636.94 & 6.64 \\
\hline 04 & 12401.48 & 12.40 & 10645.78 & 10.65 \\
\hline 05 & 7898.83 & 7.90 & 9076.17 & 9.08 \\
\hline 06 & 4317.88 & 4.32 & 2308.46 & 2.31 \\
\hline 07 & 7716.38 & 7.72 & 6742.02 & 6.74 \\
\hline 08 & 8881.74 & 8.88 & 8200.99 & 8.20 \\
\hline 09 & 6319.18 & 6.32 & 3777.43 & 3.78 \\
\hline 10 & 5227.34 & 5.23 & 4684.80 & 4.68 \\
\hline 11 & 6970.82 & 6.97 & 7570.30 & 7.57 \\
\hline 12 & 11765.66 & 11.77 & 13084.66 & 13.08 \\
\hline Total & 96531.94 & 96.53 & 92282.54 & 92.28 \\
\hline
\end{tabular}

\section{Tendencia de las precipitaciones analizadas}

El comportamiento de las precipitaciones durante los últimos 60 meses evaluados desde el año 20162020, se observa que los pronósticos a partir del mes 65 empiezan a bajar (Figura, 3). 


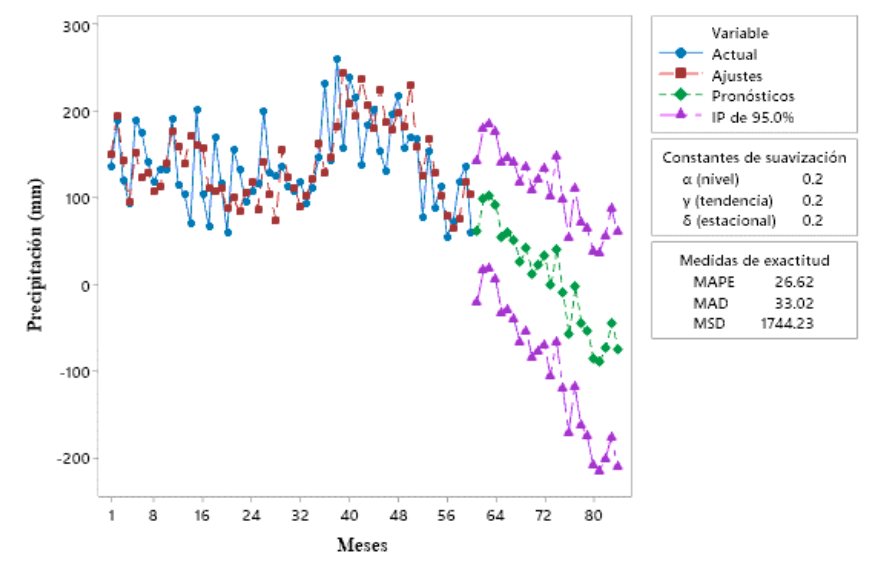

Figura 3. Comportamiento de las precipitaciones mensuales

Tabla 6, se muestra los valores de los pronósticos para los años 2021 y 2022. Para el año 2021, la precipitación anual será de 2258.057 mm, mientras que para el año 2022 la precipitación será de 2158.656 mm. Las precipitaciones al 2022 disminuirán en 99.402 mm. El límite inferior para el año 2021 es de $1148.52 \mathrm{~mm}$ y el límite superior es de $3367.58 \mathrm{~mm}$. Mientras que para el año 2022 el límite inferior de precipitación es 1031.74 mm a comparación del límite superior es 3285.57 mm.

Tabla 6. Predicción de las precipitaciones

\begin{tabular}{ccccccc}
\hline $\begin{array}{c}\text { Meses del } \\
\text { año }\end{array}$ & $\begin{array}{c}\text { Predicción de } \\
\text { las } \\
\text { precipitaciones } \\
\text { para el 2021 } \\
(\mathbf{m m})\end{array}$ & $\begin{array}{c}\text { Límite de } \\
\text { confianza } \\
\text { inferior } \\
\text { (LCI) }\end{array}$ & $\begin{array}{c}\text { Límite de } \\
\text { confianza } \\
\text { superior } \\
\text { (LCS) }\end{array}$ & $\begin{array}{c}\text { Predicción de } \\
\text { las } \\
\text { precipitaciones } \\
\text { para el 2022 } \\
\text { (mm) }\end{array}$ & $\begin{array}{c}\text { Límite de } \\
\text { confianza } \\
\text { inferior } \\
\text { (LCI) }\end{array}$ & $\begin{array}{c}\text { Límite de } \\
\text { confianza } \\
\text { superior } \\
\text { (LCS) }\end{array}$ \\
\hline 01 & 135.357 & 92.9 & 177.8 & 152.382 & 109.268 & 195.496 \\
02 & 260.199 & 96.6 & 423.8 & 305.045 & 138.841 & 471.25 \\
03 & 190.003 & 132.3 & 247.7 & 155.25 & 96.664 & 213.836 \\
04 & 290.093 & 149.9 & 430.3 & 249.024 & 106.624 & 391.425 \\
05 & 184.768 & 39.9 & 329.6 & 212.308 & 65.2194 & 359.396 \\
06 & 101.003 & 79.6 & 122.4 & 53.999 & 32.27 & 75.729 \\
07 & 180.5 & 98.7 & 262.3 & 157.708 & 74.6449 & 240.771 \\
08 & 207.76 & 52.4 & 363.1 & 191.836 & 34.0783 & 349.594 \\
09 & 147.817 & 98.4 & 197.2 & 88.361 & 38.161 & 138.561 \\
10 & 122.277 & 108.1 & 136.4 & 109.586 & 95.206 & 123.966 \\
11 & 163.06 & 53.8 & 272.3 & 177.083 & 66.085 & 288.08 \\
12 & 275.22 & 145.9 & 404.6 & 306.074 & 174.681 & 437.467 \\
\hline Total & 2258.057 & 1148.5258 & 3367.589 & 2158.656 & 1031.7426 & 3285.571 \\
\hline
\end{tabular}




\section{DISCUSIÓN}

Las precipitaciones estacionales en la región Amazonas se ha incrementado en los últimos años (Espinoza, 2009). En muchas oportunidades las precipitaciones en la zona del nor oriente peruano ha generado inundaciones, por lo general estos eventos ocurre durante los meses de febrero (temporada lluviosa) (INDECI, 2020). Esta investigación afirma que las precipitaciones máximas ocurren durante las temporadas de lluvias anuales (diciembre -abril) y las mínimas ocurre durante los meses de estiaje (junio-setiembre).

La medida de error MAPE, permite tener la certeza de la confiabilidad de los datos evaluados (Arruti, 2019). En ese sentido el MAPE más cerca a uno fue de 4.8 para el mes de octubre. El modelo aditivo Holt- Winters permitió estimar precipitaciones de 2258.057 mm para el año 2021 y para el 2022 las estimaciones indican una disminución de $100 \mathrm{~mm}$. Las estimaciones arrojan valores promedios por debajo $3121 \mathrm{~mm}$, promedios estimados durante los años 2013-2018 (Cornejo et al., 2017). Las precipitaciones son muy heterogéneos, en el nor este de Brasil, encontraron valores superiores a 2000 mm y 800 mm en la época de sequía (Catulé et al., 2018).

Las proyecciones de captación de agua de lluvia, es importante porque nos permite determinar el abastecimiento de agua por persona y estimar la cantidad de agua para una familia promedio de las zonas indígenas. El área de techo es importante para abastecer de agua a una familia, con un techo promedio de 47,5 $\mathrm{m}^{2}$ se puede aprovechar 76 361,76 litros/año. Díaz-Ortiz Medina-Tafur, (2020), encontraron que la demanda de agua por persona en comunidad nativa es de 60 litros/día. Los resultados están por encima de la norma peruana, que establece la dotación normativa de 30 1/persona/día (Ministerio de Vivienda, Construcción y Saneamiento, 2016). La cantidad de agua en una vivienda estará en función a las actividades básicas que se desarrollen, en tal sentido se recomienda, su uso el consumo humano y preparado de alimentos. Con la estimación del modelo Holt-Winters aditivo estimo, para el año 2021 $96.53 \mathrm{~m}^{3}$ de agua y pronóstico para el año 2022 fue $92.28 \mathrm{~m}^{3}$. Las predicciones de precipitaciones, pueden estar sujetas a la las condiciones climáticas futuras, al tener variaciones máximas y mínimas (Robinson et al., 2017). En ese sentido las estimaciones están a los LCI y el LCS propios del modelo Holt-Winters (Franco Rodríguez \& Velasteguí López, 2020).

Los sistemas de almacenamiento y captación de agua en comunidades nativas son importantes e incluso pueden reemplazar al agua potable (Agatón, 2016). Antes de empezar la implantación de sistemas de captación de agua de lluvia es importante realizar un análisis de las precipitaciones para diferentes escenarios (Chiu et al., 2009). En ese sentido las proyecciones de captación de agua de lluvia futura, es 
de gran utilidad para la toma de decisiones en la instalación de proyectos, en las zonas rurales de la amazonia. Las tendencias, permite determinar el comportamiento de las precipitaciones a los años futuros y ayuda a predecir deslizamiento de tierras (Orozco et al., 2011).

\section{CONCLUSIONES}

La determinación de pronósticos para datos de precipitación es importante, antes de la toma decisiones en la instalación de proyectos de captación de agua de lluvia. Con un área de techo de $47.5 \mathrm{~m}^{2}$ permitió estimar $96.53 \mathrm{~m}^{3}$ de agua de lluvia para el 2021 y para el año 2022, habrá una disminución de $4.25 \mathrm{~m}^{3}$ de agua. Sin embargo, las áreas de techo de las viviendas son variables y la captación de agua puede estar sujeta al área de cada vivienda en las zonas de estudio.

\section{AGRADECIMIENTOS}

Al proyecto de investigación "Prototipos de sistema de potabilización del agua de lluvia en comunidades nativas del departamento de Amazonas. Perú 2019-2020” (PROLLUVIA), al Instituto de Investigación para el Desarrollo Sustentable de Ceja de Selva (INDES-CES) de la Universidad Nacional Toribio Rodríguez de Mendoza de Amazonas, por el apoyo logístico para realizar la investigación, así como al FONDECYT por el financiamiento del proyecto de investigación mediante contrato de subvención $\mathrm{N}^{\circ}$ 185-2018- FONDECYT-BM-IADT-SE.

\section{REFERENCIAS BIBLIOGRÁFICAS}

Agatón, Adolfo León, Juan Carlos Córdoba RuizUriel, F. C. S. (2016). Revisión del estado de arte en captación y aprovechamientode aguas lluvias en zonas urbanas y aeropuertos. Tecnura, 20(50), 141-153. https://doi.org/10.14483/udistrital.jour.tecnura.2016.4.a10

Anggono, D., Suparjo, W., Hartomo, K. D., Tanone, R., Informasi, F. T., Studi, P., Informatika, T., Kristen, U., Wacana, S., \& Pendahuluan, I. (2018). Model Prediksi Kekeringan Menggunakan Metode Holt-Winters (Studi Kasus : Wilayah Kabupaten Boyolali).

Arruti, A. R. S. G. S. (2019). Evaluación preliminar de un sistema de pronóstico de precipitación a muy corto plazo basado en la extrapolación de datos sintéticos de radar. Meteoro Lógico, 44.

Catulé, P. F., Presidente, U., \& Carlos, A. (2018). Estudo de verificação da viabilidade de captação e uso de água da chuva no município de Teófilo Otoni - MG. c, 1-25. 
Chiu, Y. R., Liaw, C. H., \& Chen, L. C. (2009). Optimizing rainwater harvesting systems as an innovative approach to saving energy in hilly communities. Renewable Energy, 34(3), 492-498. https://doi.org/10.1016/j.renene.2008.06.016

Cornejo, M. V., Del, P., Ministros, C. D. E., Arévalo, C. V., \& Aponte, F. C. (2017). Presidente Constitucional de la República.

Dhamodharavadhani, S., \& Rathipriya, R. (2019). Region-Wise Rainfall Prediction Using MapReduceBased Exponential Smoothing Techniques. In Advances in Intelligent Systems and Computing (Vol. 750). Springer Singapore. https://doi.org/10.1007/978-981-13-1882-5_21

Díaz-Ortiz, E. A. A. M.-T. (2020). Demanda, colecta y calidad del agua de lluvia en la comunidad nativa yahuahua, nieva, amazonas (Perú). Revista de Investigación Científica REBIOL, 40(2), 188-205. Espinoza Villar Lavado; Ordoñez, Juan Julio; Fraizy, Pascal; Guyot, Jean Loup ; Ronchail, Josyane; Vauchel, Philippe; Cochonneau, Gérard; Filizola, Naziano; Labat, David;De Oliveira, Eurides, J. C. W. (2009). Evolución regional de los caudales en el conjunto de la cuenca del Amazonas para el periodo 1974-2004 y su relación con factores climáticos. Revista Peruana GeoAtmosférica RPGA, 1(1), 66-89.

Franco Rodríguez, M. del C., \& Velasteguí López, E. (2020). El suavizado exponencial en el pronóstico de series no estacionarias. Ciencia Digital, 4(1), 97-110. https://doi.org/10.33262/cienciadigital.v4i1.1073

González-Reyes, Á., \& Muñoz, A. A. (2013). Cambios en la precipitación de la ciudad de Valdivia (Chile) durante los últimos 150 años. Bosque, 34(2), 191-200. https://doi.org/10.4067/S071792002013000200008

Heydari, M., Ghadim, H. B., Rashidi, M., \& Noori, M. (2020). Application of holt-winters time series models for predicting climatic parameters (Case study: Robat Garah-Bil station, Iran). Polish Journal of Environmental Studies, 29(1), 617-627. https://doi.org/10.15244/pjoes/100496

INDECI. (2020). Precipitaciones pluviales en el departamento de Arequipa.

Liu, L., \& Wu, L. (2020). Holt-Winters model with grey generating operator and its application. Communications in Statistics - Theory and Methods, 0(0), 1-14. https://doi.org/10.1080/03610926.2020.1797804

Magallanes-Quintanar, R., Blanco-Macías, F., Galván-Tejada, E. C., Galván-Tejada, J., MárquezMadrid, M., \& Valdez-Cepeda, R. D. (2019). Negative regional standardized precipitation index 
trends prevail in the Mexico’s state of Zacatecas. Terra Latinoamericana, 37(4), 487-499. https://doi.org/10.28940/terra.v37i4.563

Manideep, K., \& Sekar, K. R. (2018). Rainfall prediction using different methods of Holt winters algorithm: A big data approach. Int. J. Pure Appl. Math, 119(15), 379-386. https://acadpubl.eu/hub/2018-119-15/1/38.pdf

Márdero, S., Nickl, E., Schmook, B., Schneider, L., Rogan, J., Christman, Z., \& Lawrence, D. (2012). Sequías en el sur de la península de Yucatán: análisis de la variabilidad anual y estacional de la precipitación. Investigaciones Geográficas, 78, 19. https://doi.org/10.14350/rig.32466

Mohammad, M., Malakoutian, A., Malakoutian, Y., Mostafapoor, P., Mohammad, M., Malakoutian, A., Malakoutian, Y., Mostafapoor, P., Ziafat, S., \& Abed, D. (2021). Prediction for Monthly Rainfall of Six Meteorological Regions and TRNC ( Case Study : North Cyprus ) To cite this version : HAL Id : hal-03228691 Prediction for Monthly Rainfall of Six Meteorological Regions and TRNC ( Case Study : North Cyprus ).

Okagbue, H. I., Oguntunde, P. E., Obasi, E. C. M., \& Akhmetshin, E. M. (2021). Trends and usage pattern of SPSS and Minitab Software in Scientific research. Journal of Physics: Conference Series, 1734(1). https://doi.org/10.1088/1742-6596/1734/1/012017

Olivares, B. O., Cortez, A., Lobo, D., Parra, R. M., Rey B., J. C., \& Rodríguez, M. F. (2016). Estudio de la Sequía Meteorológica en Localidades de los Llanos de Venezuela Mediante el Índice de Precipitación Estandarizado. Acta Nova, 7(3), 266-283.

Orozco, O. L. P., Escobar, Y. C., \& Angel, M. Q. (2011). Estudio de tendencias de la precipitación mensual en la cuenca alta-media del río Cauca, Colombia. DYNA (Colombia), 78(169), 112120.

Priyan, K. (2015). Spatial and Temporal Variability of Rainfall in Anand District of Gujarat State. Aquatic Procedia, 4(Icwrcoe), 713-720. https://doi.org/10.1016/j.aqpro.2015.02.092

Puah, Y. J., Huang, Y. F., Chua, K. C., \& Lee, T. S. (2016). River catchment rainfall series analysis using additive holt-Winters method. Journal of Earth System Science, 125(2), 269-283. https://doi.org/10.1007/s12040-016-0661-6

Pushpalatha, S. P. M. (2019). Drought prediction based on SPI and SPEI with varying timescales using LSTM recurrent neural network. Soft Computing, 1. https://doi.org/10.1007/s00500-019-041201 
Raha, S., \& Gayen, S. K. (2021). Comparative study of different exponential smoothing models in simulation of meteorological drought: A study on purulia district, west bengal, india. Journal of Applied and Natural Science, 13(2), 504-511. https://doi.org/10.31018/jans.v13i2.2637

Robinson, J. D., Vahedifard, F., \& Aghakouchak, A. (2017). Rainfall-triggered Slope Instabilities under a Changing Climate: Comparative Study using Historical and Projected Precipitation Extremes Joe D. Robinson 1, Farshid Vahedifard 2 , Amir AghaKouchak 3 1. Canadian Geotechnical Journal.

Salauddin Khan, M., Islam, M., Adhikary, S., Murad Hossain, M., \& Afroja, S. (2018). Analysis and Predictions of Seasonal Affected Weather Variables of Bangladesh: SARIMA Models vs. Traditional Models. International Journal of Business and Management, 13(12), 70. https://doi.org/10.5539/ijbm.v13n12p70

Shah, U., Garg, S., Sisodiya, N., Dube, N., \& Sharma, S. (2018). Rainfall prediction: Accuracy enhancement using machine learning and forecasting techniques. PDGC 2018 - 2018 5th International Conference on Parallel, Distributed and Grid Computing, 776-782. https://doi.org/10.1109/PDGC.2018.8745763

Siles, G., \& Chirinos, D. (2018). Estimación De Intensidad De Lluvia Para Estudios De Propagación Radioeléctrica En Bolivia. Investigacion \& Desarrollo, 18(1), 69-79. https://doi.org/10.23881/idupbo.018.1-5i

Sinay, L. J., \& Kembauw, E. (2021). Monthly Rainfall Components in Ambon City : Evidence from the Serious Time Analysis Monthly Rainfall Components in Ambon City : Evidence from the Serious Time Analysis. https://doi.org/10.1088/1755-1315/755/1/012079

Sopipan, N. (2014). Forecasting Rainfall in Thailand: A Case Study of Nakhon Ratchasima Province. 8(11), 712-716.

Tabari, H., Abghari, H., \& Talaee, P. H. (2011). Temporal trends and spatial characteristics of drought and rainfall in arid and semiarid regions of Iran. https://doi.org/10.1002/hyp

Wichitarapongsakun, P., Sarin, C., Klomjek, P., \& Chuenchooklin, S. (2016). Rainfall prediction and meteorological drought analysis in the Sakae Krang River basin of Thailand. Agriculture and Natural Resources, 50(6), 490-498. https://doi.org/10.1016/j.anres.2016.05.003

Wong, C. L. (2009). Variability of rainfall in Peninsular Malaysia. 5471-5503. 